\title{
Cellular identity of an 18S rRNA gene sequence clade within the class Kinetoplastea: the novel genus Actuariola gen. nov. (Neobodonida) with description of the type species Actuariola framvarensis sp. nov.
}

\author{
Thorsten Stoeck, ${ }^{1}$ M. V. Julian Schwarz, ${ }^{1}$ Jens Boenigk, ${ }^{2}$ \\ Michael Schweikert, ${ }^{3}$ Sophie von der Heyden ${ }^{4}$ and Anke Behnke ${ }^{1}$ \\ ${ }^{1}$ Department of Biology, TU Kaiserslautern, Erwin-Schrödinger Str. 14, D-67663 Kaiserslautern, \\ Germany \\ ${ }^{2}$ Austrian Academy of Sciences, Institute for Limnology, Mondseestr. 9, A-5310 Mondsee, \\ Austria \\ ${ }^{3}$ Institute of Biology, University Stuttgart, Pfaffenwaldring 57, D-70550 Stuttgart, Germany \\ ${ }^{4}$ Department of Zoology, University of Oxford, South Parks Road, Oxford OX1 3PS, UK
}

Correspondence

Thorsten Stoeck

stoeck@rhrk.uni-kl.de

\begin{abstract}
Environmental molecular surveys of microbial diversity have uncovered a vast number of novel taxonomic units in the eukaryotic tree of life that are exclusively known by their small-subunit (SSU) rRNA gene signatures. In this study, we reveal the cellular and taxonomic identity of a novel eukaryote SSU rRNA gene sequence clade within the Kinetoplastea. Kinetoplastea are ubiquitously distributed flagellated protists of high ecological and medical importance. We isolated an organism from the oxic-anoxic interface of the anoxic Framvaren Fjord (Norway), which branches within an unidentified kinetoplastean sequence clade. Ultrastructural studies revealed a typical cellular organization that characterized the flagellated isolate as a member of the order Neobodonida Vickerman 2004, which contains five genera. The isolate differed in several distinctive characters from Dimastigella, Cruzella, Rhynchobodo and Rhynchomonas. The arrangement of the microtubular rod that supports the apical cytostome and the cytopharynx differed from the diagnosis of the fifth described genus (Neobodo Vickerman 2004) within the order Neobodonida. On the basis of both molecular and microscopical data, a novel genus within the order Neobodonida, Actuariola gen. nov., is proposed. Here, we characterize its type species, Actuariola framvarensis sp. nov., and provide an in situ tool to access the organism in nature and study its ecology.
\end{abstract}

\section{INTRODUCTION}

Analysis of small-subunit (SSU) rRNA genes (rRNA approach) has been a valuable tool for revealing the diversity of eukaryotic micro-organisms in many different environments (Amaral Zettler et al., 2002; López-García et al., 2001, 2003; Stoeck et al., 2003a; Willerslev et al., 1999). SSU rRNA genes directly amplified from environmental samples have demonstrated a vast number of eukaryote lineages ranging from putatively novel species to suggested

Published online ahead of print on 1 July 2005 as DOI 10.1099/ ijs.0.63769-0.

Abbreviation: SSU, small-subunit.

The GenBank/EMBL/DDBJ accession number for the SSU rRNA gene sequence of Actuariola framvarensis strain FV18-8TS is AY963571. novel kingdoms (Berney et al., 2004; Cavalier-Smith, 2004; Dawson \& Pace, 2002). However, many of these newly discovered groups are only known from their molecular signatures. Because the rRNA approach provides little information beyond the fact of an organism's existence, distribution in nature and molecular phylogeny, nothing is revealed about the organism's cellular identity, ecological role, in situ abundance and physiological capacities.

Examples of such putatively novel phylogenetic lineages can be found in various taxonomic levels throughout the eukaryotic tree of life, for example, among the Kinetoplastida. Kinetoplastida are protozoan organisms that probably diverged early in evolution from other eukaryotes (Moreira et al., 2004). They are characterized by a number of unique features with respect to their energy and carbohydrate 
metabolism (Hannaert et al., 2003). Kinetoplastids include disease-causing parasites such as Trypanosoma spp. and Leishmania spp. as well as free-living forms of ecological importance in terrestrial and aquatic ecosystems, commonly known as bodonids (Arndt et al., 2000; Foissner, 1991). Until recently, little was known about their evolution and ecology (Callahan et al., 2002; Dyková et al., 2003; Moreira et al., 2004; Simpson et al., 2002).

Kinetoplastids belong, together with euglenids and diplonemids, to the phylum Euglenozoa (Cavalier-Smith, 1981) and are grouped in the class Kinetoplastea. Recently, Moreira et al. (2004) updated kinetoplastid phylogeny using environmental sequences, and proposed a revised classification. At about the same time, a bodonid sequence was published (von der Heyden et al., 2004) which, together with an environmental sequence (López-García et al., 2003; Moreira et al., 2004), confirmed the existence of an as yet undescribed sequence clade within the order Neobodonida Vickerman 2004. Meanwhile, further $18 \mathrm{~S}$ rRNA gene analyses verified and strengthened this sequence clade, which appears to consist of free-living organisms from aquatic as well as terrestrial habitats (von der Heyden, 2004). However, no cultured representative of this clade has been reported. Thus, its morphological, ultrastructural, ecological and physiological identity is unknown.

Within the framework of a diversity survey using molecular and culturing approaches, we succeeded in isolating and culturing an organism from suboxic fjord water (Norway), which, according to its phylogenetic position, branches within this undescribed neobodonid sequence clade. Here we elucidate its cellular identity, taxonomic state and some ecophysiological capacities, and provide a tool to access this organism in nature.

\section{METHODS}

Sampling and enrichment. Samples were collected from the Framvaren Fjord, Norway. The fjord is characterized by a chemocline at a depth of about $18 \mathrm{~m}$, with anoxic conditions below the chemocline (Skei, 1988). For cultivation, samples were taken from the oxic-anoxic interface $(18 \mathrm{~m})$ in the central basin of the fjord using 51 Niskin bottles. Water was withdrawn from the bottles with a $50 \mathrm{ml}$ syringe attached to the bottle's outlet. For oxic enrichment, $50 \mathrm{ml}$ sample water was added to $100 \mathrm{ml}$ modified FøynsErdschreiber medium $(26 \%$, pH $7 \cdot 5$; CCAP) or artificial sea water (ASW; $26 \%$, pH 7.5) in $200 \mathrm{ml}$ glass vials, and $0 \cdot 2 \%(\mathrm{v} / \mathrm{v})$ glycerol and $0 \cdot 1 \%(\mathrm{w} / \mathrm{v})$ yeast extract were added to support growth of bacteria. The samples were incubated at room temperature and at $12{ }^{\circ} \mathrm{C}$. The first increase in protistan abundance could be observed after 7 days.

Isolation of the flagellate strain. The basal medium for the cultivation and isolation of the flagellate strain was an inorganic basal medium containing $\left(\mathrm{g} \mathrm{l}^{-1}\right): \mathrm{K}_{2} \mathrm{HPO}_{4} .3 \mathrm{H}_{2} \mathrm{O}, 0 \cdot 007 ; \mathrm{KNO}_{3}, 0 \cdot 07$; $\mathrm{CaCl}_{2} .2 \mathrm{H}_{2} \mathrm{O}, 1 \cdot 015 ; \mathrm{MgSO}_{4} .7 \mathrm{H}_{2} \mathrm{O}, 4 \cdot 844 ; \mathrm{MgCl}_{2} \cdot 6 \mathrm{H}_{2} \mathrm{O}, 3 \cdot 857 ; \mathrm{KCl}$, $0 \cdot 469$; and $\mathrm{NaCl}, 19 \cdot 705$. The isolation protocol followed the approach of Boenigk et al. (2005). The original sample was diluted to an abundance of 0.5 flagellates $\mathrm{ml}^{-1}$, transferred to 24 -well cell culture plates ( $1 \mathrm{ml}$ per well) and supplemented with various food sources, i.e. heat-killed cultures of bacterial strain MWH-Mol (class
Actinobacteria; Hahn et al., 2003) or Listonella pelagia CB5 at a con-

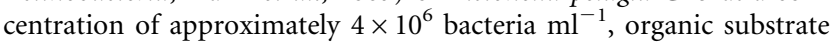
(nutrient broth, soyotone peptone and yeast extract) at various final concentrations, or no food addition. The wells were checked microscopically for positive growth every second day for a period of at least 2 weeks. When flagellate growth was detected, the medium was transferred to a $50 \mathrm{ml}$ Erlenmeyer flask containing inorganic basal medium and fresh food bacteria. After 2-6 days, the subsamples were further diluted to final concentrations of $0 \cdot 05,0 \cdot 1,0 \cdot 2$ and $0 \cdot 4$ flagellates $\mathrm{ml}^{-1}$ and supplemented with the bacterial strain $L$. pelagia CB5 as described above. Each of these dilutions were transferred to wells of sterile 24 -well cell culture plates $(1 \mathrm{ml}$ per well) and incubated at $22^{\circ} \mathrm{C}$. Screening of the wells for growth of flagellates was again performed by direct microscopy every second day. This procedure was repeated until pure cultures were established. Pure cultures were acclimatized to $16^{\circ} \mathrm{C}$ and transferred to permanent culture (inorganic basal medium supplemented with wheat grain in $50 \mathrm{ml}$ cell culture flasks, at $16^{\circ} \mathrm{C}$ and in low light; subcultured in fresh medium with wheat grain once per month).

DNA extraction and PCR amplification. DNA from cultures was extracted using the DNEasy tissue kit (Qiagen). A $1 \mathrm{ml}$ aliquot of the culture (approximately 5000 cells) was withdrawn on a $0.65 \mu \mathrm{m}$ Durapore filter (Millipore) under gentle vacuum. The filter was then incubated with ATL buffer (Qiagen) and the extraction followed the protocol of the manufacturer for animal tissues. The SSU rRNA gene was PCR-amplified first with the general eukaryotic primers EukA (5'-AACCTGGTTGATCCTGCCAGT-3') and EukB (5'-TGATCCTTCTGCAGGTTCACCTAC-3') and, after initial phylogenetic analysis, again with the kinetoplastid-specific primers Kineto14F (5'-CTGCCAGTAGTCATATATGCTTGTTTCAAGGA-3') and Kineto2026R (5'-GATCCTCTGCAGGTTCACCTACAGCT-3') (von der Heyden et al., 2004). The PCR protocol employed HotStart Taq DNA polymerase (Qiagen) and consisted of an initial hot-start incubation $\left(15 \mathrm{~min}\right.$ at $95^{\circ} \mathrm{C}$ ) followed by 30 identical amplification cycles (denaturation at $95^{\circ} \mathrm{C}$ for $45 \mathrm{~s}$, annealing at $55^{\circ} \mathrm{C}$ with EukA-EukB primers or $65^{\circ} \mathrm{C}$ with the kinetoplastid-specific primers for $1 \mathrm{~min}$, and extension at $72{ }^{\circ} \mathrm{C}$ for $2.5 \mathrm{~min}$ ) and final extension at $72{ }^{\circ} \mathrm{C}$ for $7 \mathrm{~min}$. The PCR products were cloned using the pGEM$\mathrm{T}$ Vector System II cloning kit (Promega). Plasmids were isolated from overnight cultures by using a Qiagen Plasmid Mini kit and several clones were sequenced bidirectionally by MWG-Biotech. The sequences obtained from several clones were almost identical (sequence similarity $99 \cdot 7 \%$ ).

Phylogeny and sequence analysis. To evaluate the approximate phylogenetic position of the target organism, we compiled its SSU rRNA gene sequence in ARB (Ludwig et al., 2004) and aligned the sequence with $>5000$ prealigned eukaryotic sequences using the ARB fast Aligner utility. The alignment was manually refined according to phylogenetically conserved secondary structures. Using the Quickadd-Parsimony tool of ARB, we evaluated the approximate phylogenetic position of the sequence. For greater resolution, the target sequence was then aligned with almost all available apical bodonid sequences $(n=26)$, and additionally with seven apical kinetoplastid sequences (four trypanosomatids and three prokinetoplastids as an outgroup; Moreira et al., 2004). The sequences were aligned using CLUSTAL_X (Thompson et al., 1994) and manually adjusted using MacClade version 4.06 (Maddison \& Maddison, 2000). Nonconserved positions were excluded from the phylogenetic analyses, resulting in a dataset of 1404 unambiguously aligned positions. A maximum-likelihood tree and an evolutionary distance tree under maximum-likelihood criteria were constructed under the general time-reversible (GTR) model using PAUP ${ }^{*}$ software package $4.0 \mathrm{~b} 10$ (Swofford, 2001). We allowed for rate variation across sites, assuming a gamma distribution $(0 \cdot 6043)$ and a proportion of invariable sites $(0 \cdot 4047)$ estimated by using MODELTEST (Akaike information 
criterion; Posada \& Crandall, 1998). Base frequencies as determined by using MODELTEST for A, C, G and T were $0 \cdot 2691,0 \cdot 2098,0 \cdot 2770$ and $0 \cdot 2441$, respectively, with the rate matrix of the substitution model being $1.2126(\mathrm{~A}-\mathrm{C}), 2 \cdot 1087(\mathrm{~A}-\mathrm{G}), 1.6535(\mathrm{~A}-\mathrm{T}), 0.7491$ $(\mathrm{C}-\mathrm{G}), 5 \cdot 6137(\mathrm{C}-\mathrm{T})$ and $1 \cdot 0(\mathrm{G}-\mathrm{T})$. We assessed the relative stability of the tree topology by using 1000 distance bootstrap replicates and 100 maximum-likelihood bootstrap replicates. The settings for bootstrap calculations were the same as those given above.

Sequence similarities were calculated based on conserved secondary structures only (1404 aligned positions). Variable regions were excluded from similarity calculations, because the mean similarity within the same species (e.g. Neobodo designis, four sequences available in GenBank, accession nos AY490235, AF464896, AY425016 and AF209856; Doležel et al., 2000; von der Heyden et al., 2004; X. Gu, Y. Yu $\&$ Y. Shen, unpublished data) varies considerably when the complete sequence is taken into account (mean similarity $91 \cdot 88 \pm 1 \cdot 08 \% ; n=6$ ). Thus, similarity values between species are of limited value when considering the complete sequence, variable regions included. We used the PAUP* software package 4.0b10 (Swofford, 2001) to calculate sequence similarities.

Microscopy. Light microscopic observations were made with a Zeiss Axiophot II in brightfield, phase and interference contrast, in part after immobilization in low-melting-point agarose (Reize \& Melkonian, 1989). For FITC (fluorescein isothiocyanate)-DAPI (4',6-diamidino-2-phenylindole) double staining, cells were fixed with alkaline Lugol solution $(0 \cdot 1 \%$ final concentration), particlefree formaldehyde $(1 \cdot 8 \%)$ and sodium thiosulfate $\left(60 \mathrm{~g} \mathrm{ml}^{-1}\right)$ (modified from Del Giorgio et al., 1996) at $4{ }^{\circ} \mathrm{C}$ for $1 \mathrm{~h}$. Subsamples were concentrated on black polycarbonate membranes (Millipore type ATTP; diameter, $25 \mathrm{~mm}$; pore size, $0.8 \mu \mathrm{m}$ ) and stained first with DAPI $\left(1 \mathrm{~g} \mathrm{ml}^{-1} ; 3 \mathrm{~min}\right)$ and then with FITC $\left(33 \mathrm{~g} \mathrm{ml}^{-1}\right.$; $6 \mathrm{~min})$. The filters were mounted on microscope slides and cells were visualized by epifluorescence microscopy at different magnifications. Cells could be unambiguously identified by a combined inspection at UV and blue excitation (filter sets Zeiss01 and Zeiss09, respectively) to detect both their DAPI-stained nucleus and kinetoplast and their FITC-stained body outline with the flagella.

For scanning electron microscopy (SEM), cells in culture medium from exponential growth phase were fixed with $2 \cdot 5 \%$ glutaraldehyde (final) for $1 \mathrm{~h}$ at $4{ }^{\circ} \mathrm{C}$ and drawn onto a polycarbonate filter $(24 \mathrm{~mm}, 0 \cdot 8 \mu \mathrm{m})$. After rinsing the filter with $4 \mathrm{ml} 1 \times \mathrm{PBS}$, the cells were post-fixed on the filter with $1 \% \mathrm{OsO}_{4}$ in $0 \cdot 1 \mathrm{M}$ cacodylate buffer for $1 \mathrm{~h}$ at room temperature, gently rinsed with $0 \cdot 1 \mathrm{M}$ cacodylate buffer and taken through a graded ethanol dehydration before being chemically dried with hexamethyldisilazane (HMDS) (Stoeck et al., 2003b). The dry filter was quartered and each piece was mounted on an aluminium stub. The stubs were coated with gold (Edwards E306) and observed with a Zeiss DSM940.

For transmission electron microscopy (TEM), cells were fixed in $2.5 \%$ glutaraldehyde in ASW for $1 \mathrm{~h}$, washed several times with this medium, post-fixed in $1 \% \mathrm{OsO}_{4}$ (in ASW) for $1 \mathrm{~h}$ and dehydrated in an acetone series $(30,50,75,90,100$ and $100 \%)$ for $20 \mathrm{~min}$. Finally, cells were embedded in Spurr's resin (Spurr, 1969). Ultrathin sections were obtained with a Leica UCT Ultracut, and post-stained with lead citrate (Reynolds, 1963) for $4 \mathrm{~min}$ and $1 \%$ aqueous uranyl acetate for $2 \mathrm{~min}$. For whole-mount preparation, cells were fixed in $2.5 \%$ glutaraldehyde for $30 \mathrm{~min}$ and a drop was transferred directly onto a pioloform-coated copper grid. After about $10 \mathrm{~min}$, the liquid was removed with filter paper and a drop of $1 \%$ aqueous uranyl acetate was added. The liquid was removed and the preparation was allowed to air dry. All TEM observations were done using a Zeiss EM 10 at $60 \mathrm{kV}$.

Ecophysiological tolerance limits. Ecophysiological tolerance limits were tested using 12 -well cell culture plates. Test medium
(4 ml) supplemented with food bacteria $\left(15 \times 10^{6}-25 \times 10^{6}\right.$ bacteria $\mathrm{ml}^{-1}$ of heat-killed L. pelagia CB5) and $200 \mu$ l flagellate culture were transferred into each well, yielding the following final test conditions: salinity of $68 \cdot 4,66 \cdot 7,63 \cdot 3,59 \cdot 9,51 \cdot 4,42 \cdot 9,34 \cdot 4,17 \cdot 4,8 \cdot 9$, $7 \cdot 2,5 \cdot 7,5 \cdot 5,4 \cdot 8,4 \cdot 7,3 \cdot 8,2 \cdot 1$ and $1 \cdot 1 \mathrm{~g}^{-1} ; \mathrm{pH}$ of $10 \cdot 10,9 \cdot 70$, $9 \cdot 50,9 \cdot 03,8 \cdot 90,8 \cdot 70,6 \cdot 50,6 \cdot 00,5 \cdot 50,5 \cdot 00,4 \cdot 62,4 \cdot 22$ and $3 \cdot 80$; temperature of $4,6,8,16,22,28,30 \cdot 5$ and $31 \cdot 2{ }^{\circ} \mathrm{C}$. For temperature experiments, unaltered basal medium was used; for the salinity tolerance, media with different total amounts of salts but the same relative composition were prepared. For the $\mathrm{pH}$ experiments, we used basal medium buffered with $10 \mathrm{mM} \mathrm{Na}_{2} \mathrm{CO}_{3}$ (high pH) and $10 \mathrm{mM}$ EDTA (low $\mathrm{pH}$ ). The experimental treatments were checked every day until growth was detected, for a maximum period of 7 days. In addition to direct transfer, flagellates were stepwiseadapted to increasing/decreasing salinity and $\mathrm{pH}$. During adaptation, flagellates were allowed to grow for $48 \mathrm{~h}$ (approximately 8-12 generations) before transfer to the next test medium.

To determine the ability of the test organisms to grow under oxygen depletion, exponential-phase cells were incubated in modified FøynsErdschreiber medium $(26 \%$, $\mathrm{pH} 7 \cdot 5)$ using heat-inactivated bacteria as the primary food source. Incubations were set up in 1, 2 and $21 \%$ oxygen in the headspace $\left(\mathrm{N}_{2} / \mathrm{O}_{2}\right.$ atmosphere) and anoxically $\left(\mathrm{N}_{2}\right.$ atmosphere), at $20^{\circ} \mathrm{C}$ in the dark. Anoxic conditions were generated by adding $5 \mathrm{ml}$ sulfide $\left(0 \cdot 1 \mathrm{mg} \mathrm{Na} \mathrm{S} \mathrm{ml}^{-1}\right)$ or Anaerocult A plates (Merck) to the incubation vessels. Each incubation vessel (1 1 Schott bottles, gas-tight sealed with chlorbutyl stoppers and screw caps) contained six $10 \mathrm{ml}$ injection bottles (Ochs Glasgerätebau) inoculated with $5 \mathrm{ml}$ culture. The headspace gas was exchanged daily, with the exception of the anoxic incubations. Cell numbers were determined after 24, 48, 72 and $96 \mathrm{~h}$. After gentle shaking, two aliquots (10 $\mu \mathrm{l} \mathrm{each)}$ per sample were withdrawn from the incubation vials and counted in a Neubauer chamber. Flagellates were counted directly in six parallels each at $\times 50-100$ magnification (phase-contrast) using a Zeiss Axiophot II.

Fluorescence in situ hybridization (FISH): probe design and testing. By using the Probe_Design tool of ARB (Ludwig et al., 2004), specific oligonucleotide probes were constructed. The probes were double-checked against the GenBank dataset using BLAST search for short, almost exact matches (Altschul et al., 1997). The probe purchased (MWG Biotech) was FV18TS-650 (5'TGTCGTGCGAACCCATAG- $3^{\prime}$ ), labelled with the indocarbocyanine dye $\mathrm{Cy} 3$ at the $5^{\prime}$-end. The target position of the probe is bases 650-668 in the secondary structure of the SSU rRNA gene sequence of the isolates. The probe showed at least 2 mismatches with known higher organisms (Oryza sativa) and at least 4 mismatches with known sequenced protists available in public databases. For probe testing by fluorescence microscopy, we followed standard procedures as described elsewhere (Pernthaler et al., 2001). In short, cells in culture medium from exponential growth phase were fixed with $2.5 \%$ formaldehyde (final) for $1 \mathrm{~h}$ at $4{ }^{\circ} \mathrm{C}$ and drawn onto a black polycarbonate filter $(24 \mathrm{~mm}, 0.8 \mu \mathrm{m}$; Millipore). After rinsing the filter with $4 \mathrm{ml} 1 \times$ PBS, cells were dehydrated with a graded ethanol series and allowed to air-dry. The filters were incubated at $46^{\circ} \mathrm{C}$ for $2 \mathrm{~h}$ with a hybridization mixture containing $5 \mathrm{ng} \mu \mathrm{l}^{-1}$ (final) of the probe. The hybridization mixture was prepared as described by Pernthaler et al. (2001) with deionized formamide (stepwise increase of concentration from 0 to $90 \%$ ). Following the incubation, the filters were transferred into a preheated washing buffer (Pernthaler et al., 2001) and incubated at $48^{\circ} \mathrm{C}$ for $15 \mathrm{~min}$. After being rinsed with water and then with ethanol $(80 \%)$, the filters were air-dried and counterstained with DAPI. Fluorescence microscopy (Zeiss Axiophot II) was performed with Cy3- and DAPI-specific filter sets. Images of cells post-hybridization were captured using a cooled CCD camera (MicroCam Imager/Quanten EFF; Intas) connected to a Macintosh G5 computer. The configuration of the microscope and 
the camera remained constant throughout all experiments and all images were captured using the same exposure settings. As negative controls, we used no-probe samples, nonsense-probe samples and a non-target organism (Paramecium caudatum), together with the species-specific FV18TS-650 probe.

\section{RESULTS AND DISCUSSION}

Within the framework of a biodiversity study of the supersulfidic, anoxic Framvaren Fjord in Norway, we succeeded in isolating and culturing a flagellated organism (Fig. 1). As molecular phylogenetic and morphological/ultrastructural analyses provide the most complete and precise description of an organism, we applied both approaches to study the taxonomic identity of the isolated flagellate. Both datasets strongly suggest that the isolated organism belongs to a novel genus within the order Neobodonida Vickerman 2004 (Moreira et al., 2004), for which we propose the name Actuariola framvarensis gen. nov., sp. nov.

\section{Phylogeny}

The phylogenetic tree shows that the sequence FV188TS belongs to a strongly supported clade (AT5-25, Cryptaulaxoides-like sp. TCS2003, FV18-8TS) branching within the subclass Metakinetoplastina Vickerman 2004, class Kinetoplastea Honigberg 1963 emend. Vickerman 1976 (Fig. 2). Four different orders characterize the subclass: Eubodonida Vickerman 2004, Parabodonida Vickerman 2004, Trypanosomatida Kent 1880 stat. nov. Hollande 1952, and Neobodonida Vickerman 2004. The sequence FV188TS clearly branches within the order Neobodonida, which includes the most recent common ancestors of Neobodo Vickerman 2004 (Rhynchobodo, Rhynchomonas, Dimastigella and Cruzella), all of which are more or less well-known and described groups of organisms (Breunig et al., 1993; Eyden, 1977; Frolov \& Malysheva, 2002; Swale, 1973; Vørs, 1992). However, according to the phylogenetic analyses, the sequence clade with the sequence FV18-8TS cannot be assigned to any one of these groups, and represents a novel, strongly supported (bootstrap 97\%) taxonomic unit. The other two sequences within the clade are AT5-25, an environmental sequence of unknown morphology from a marine hydrothermal vent (López-García et al., 2003), and the sequence Cryptaulaxoides-like sp. TCS2003 that originated from a strain isolated from soil in Costa Rica (von der Heyden et al., 2004). Unfortunately, this strain is not now available and lacks a detailed morphological description. However, from what is known (T. CavalierSmith and B. Oates, unpublished data, in von der Heyden et al., 2004), its assignment to the kinetoplastids seems to be fully supported (von der Heyden et al., 2004). The mean sequence similarity within this undescribed sequence clade (FV18-8TS, AT5-25, Cryptaulaxoides-like sp. TCS2003) is $97 \cdot 83 \pm 0 \cdot 23 \%(n=3)$. This is similar to the sequence similarity between described species of well-characterized genera within the order Neobodonida, such as Neobodo $(97 \cdot 6 \pm 0.96 \%, \quad n=10 ; \quad$ GenBank accession numbers AF209856, AF464896, AJ130868, AF174379 and AF174380).
However, the closest named species to FV18-8TS is $N$. designis DH, genus Neobodo, order Neobodonida (96.43\% sequence similarity). Similarly close is the sequence of Bodo edax (genus Bodo, order Eubodonida Vickerman 2004) with $96.36 \%$ sequence similarity, followed by Cruzella marina (genus Cruzella de Faria 1922) with $95 \cdot 65 \%$ similarity to FV18-8TS. As a comparison, the sequence similarity among the closest related sequences from different genera within the same order is $96.00 \%(N$. designis $\mathrm{DH}$ and $C$. marina). B. edax is $96 \cdot 07 \%$ similar to $N$. designis $\mathrm{DH}$. Thus, the order of magnitude that distinguishes FV18-8TS from its next closest relatives in well-described genera is the same as that between the closest species of two different established genera or even orders. The phylogenetic independence of the discussed sequence clade (sequence FV18-8TS excluded) has already been shown by Simpson et al. (2002), LópezGarcía et al. (2003) and Moreira et al. (2004). Our phylogenetic analysis seems to support the monophyly of the trypanosomatids and the bodonids $70 \%$ bootstrap value in a distance analysis and $74 \%$ in a maximumlikelihood analysis), which is a controversial topic (Moreira et al., 2004). Although the statistical support is low (59\% bootstrap support in a distance analysis and unresolved in a maximum-likelihood analysis), the eubodonids seem to be sisters to the parabodonids, as also reported previously (Moreira et al., 2004; von der Heyden et al., 2004).

\section{Ultrastructure}

The ultrastructure of the isolated flagellate clearly does not match the diagnosis of any of the three orders Parabodonida Vickerman 2004 (bodonid clade 2 sensu Simpson et al. 2002), Eubodonida Vickerman 2004 (bodonid clade 3 sensu Simpson et al. 2002) or Trypansomatida Kent 1880 stat. nov. Hollande 1952 within the subclass Metakinetoplastina. Parabodonida and Eubodonida can both be diagnosed by the presence of an anterolateral cytostome, the latter also having a non-tubular, hair-bearing anterior flagellum (Moreira et al., 2004). The described isolate has an apical cytostome (Fig. 3a, d) and both flagella lack hairs (Fig. 3b), except at a short region inside the flagellar pocket (Fig. 3e). Trypanosomatida includes uniflagellated, osmotrophic parasitic species only (Moreira et al., 2004). The isolate matches the diagnosis of the order Neobodonida (bodonid clade 1 sensu Simpson et al. 2002) (Moreira et al., 2004) exactly, which is supported by the molecular $18 \mathrm{~S}$ rDNA data.

Five genera have been assigned to the order Neobodonida, Neobodo Vickerman 2004, Rhynchomonas, Dimastigella, Rhynchobodo Lackey 1940 emend. Vørs 1992, and Cruzella de Faria 1922 (Moreira et al., 2004; Vickerman, 1976). The type of kinetoplast DNA (kDNA) is an important feature in the taxonomy and phylogeny of these bodonids. The novel isolate contains prokinetoplast DNA (pro-kDNA), minicircles condensed into a single massive stainable globular bundle, located near the basal body of the flagellum (Figs 1e and $3 \mathrm{c}, \mathrm{f}, \mathrm{g}$ ), as described for the eubodonid Bodo saltans (Lukeš et al., 2002). Other neobodonids that may contain this type of kDNA include Rhynchomonas and Neobodo 


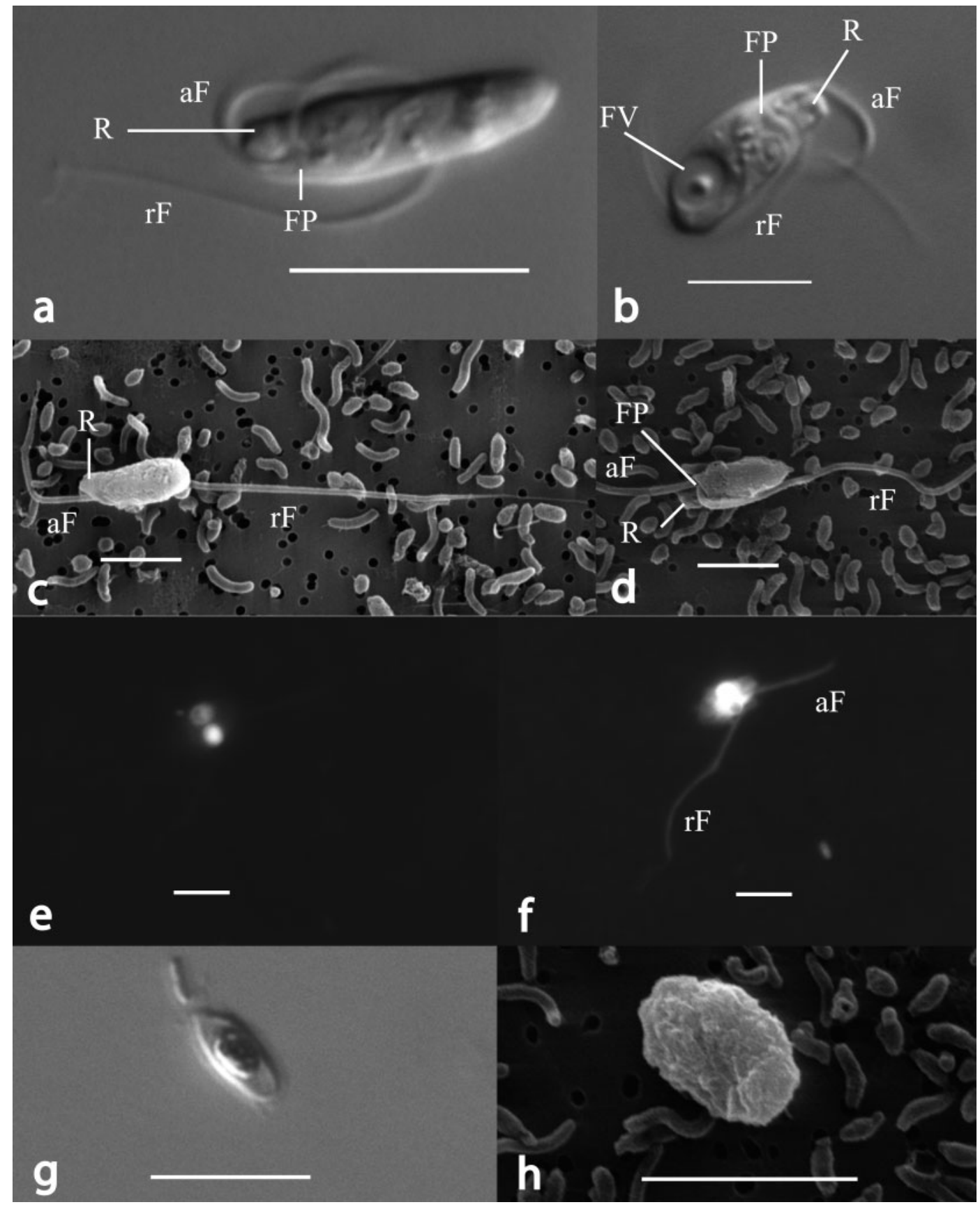

Fig. 1. Morphology of $A$. framvarensis. (a, b) Light microscopy of living cells, displaying the flagellar pocket (FP) with the anterior and recurrent flagella ( $\mathrm{aF}$ and $\mathrm{rF}$, respectively) leaving the $\mathrm{FP}$ at an angle of about $90^{\circ}$ to each other. The anterior part of the cell is characterized by a short rostrum (R). A single large food vacuole (FV) is evident in the posterior part of the cell. (c, d) SEM images of the dorsal (c) and ventral (d) views of the cell with the deep flagellar pocket (FP). The tapering ends of the flagella are evident. (e) DAPI staining of the cell's nucleus and kinetoplast. (f) FITC staining of the body outline and flagella of the same cell as in (e). The tapering end of the $\mathrm{rF}$ is visible. $(\mathrm{g}, \mathrm{h})$ Light microscopy $(\mathrm{g})$ and SEM $(\mathrm{h})$ images of cysts forming under, for example, anoxic conditions. Bars, $5 \mu \mathrm{m}$.

(Lukeš et al., 2002). The remaining neobodonid genera, Rhynchobodo, Dimastigella and Cruzella, contain distinct polykinetoplastic members (poly-kDNA) with the mitochondrial DNA arranged in monomeric minicircles distributed among various discrete loci throughout the mitochondrial lumen (Lukeš et al., 2002). The Parabodonida, e.g. Bodo sorokini (=Procryptobia sorokini), may also contain pro-kDNA, as found in our isolate from 




Fig. 2. Evolutionary distance tree under maximum-likelihood criteria of kinetoplastean SSU rRNA gene sequences showing the position of the sequence of $A$. framvarensis (FV18-8TS) and the novel neobodonid sequence clade. The tree was constructed by using a GTR + I+G DNA substitution model with the variable-site gamma distribution shape parameter (G) at 0.6043 , the proportion of invariable sites at 0.4047 and base frequencies and a rate matrix for the substitution model as determined by using MODELTEST (see Methods), based on 1404 unambiguously aligned positions. Numbers at nodes are bootstrap values. The first number shows distance bootstrap values over $50 \%$ from an analysis of 1000 bootstrap replicates and the second number maximum-likelihood bootstrap values over $50 \%$ from an analysis of 100 bootstrap replicates. Parameters for bootstrap calculations were the same as for tree construction. 


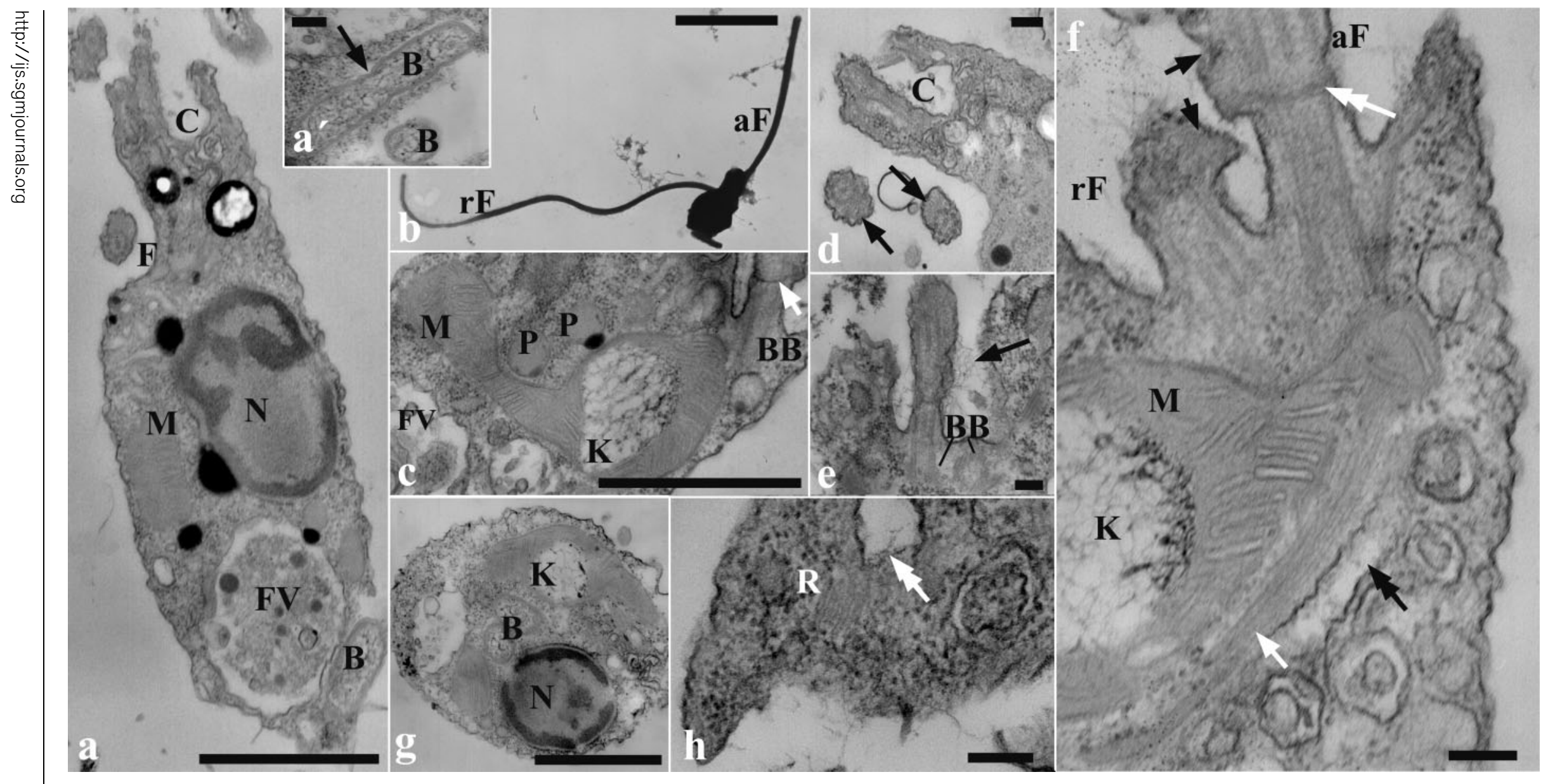

Fig. 3. TEM images of $A$. framvarensis. (a) Oblique section showing the general organization of the cell. Abbreviations: $\mathrm{B}$, intracytoplasmic bacteria; $\mathrm{C}$, cytostome; $\mathrm{F}$, flagellum; FV, food vacuole; M, mitochondrion; N, nucleus. ( $\left.a^{\prime}\right)$ Intracytoplasmic bacteria (B) in longitudinal and cross-section. One bacterium might be in an early stage of cell division (arrow). Intracytoplasmic bacteria of $A$. framvarensis are not surrounded by a peribacterial membrane. (b) Whole-mount image of $A$. framvarensis. The anterior $(\mathrm{aF})$ and the recurrent $(\mathrm{rF})$ flagella are naked. (c) The single mitochondrion $(\mathrm{M})$ encloses a kinetoplast $(\mathrm{K})$ of the pro-kDNA type and is closely associated with the basal bodies of the flagellar apparatus (BB), transitional plate (white arrow). Peroxisome-like bodies (P) are present near the mitochondrion. (d) Both flagella display a paraflagellar rod (arrows). The recurrent flagellum remains unattached to the plasma membrane. The cytostome (C) of the cell is located apically. (e) Basal bodies (BB) are arranged at an angle of about $90^{\circ}$ to each other. Fine flagellar hairs (arrow) are attached to one flagellum, restricted to the region inside the flagellar pocket. ( $f$ ) Arrangement of basal bodies with respect to the mitochondrion $(\mathrm{M})$ with the pro-kDNA type kinetoplast $(\mathrm{K})$. A bundle of microtubules representing the rod organ (white arrow) and the cytopharynx (black double arrow) follow the boundary of the mitochondrion. Both flagella ( $\mathrm{rF}$ and aF) display a paraflagellar rod (black arrows), transitional plate (white double arrow). (g) Nucleus $(\mathrm{N})$ with peripherally located heterochromatin, intracytoplasmic bacteria $(\mathrm{B})$ and the mitochondrion with kinetoplast (K). (h) Arrangement of the microtubules of the rod organ $(R)$ and the cytopharynx (white double arrow) in cross-section. The arrangement of microtubules is not triangular or prismatic. Bars, $1 \mu \mathrm{m}\left(\mathrm{a}, \mathrm{a}^{\prime}\right.$, $b, c, g)$ and $0 \cdot 1 \mu \mathrm{m}(\mathrm{d}, \mathrm{e}, \mathrm{f}, \mathrm{h})$. 
Norway (Lukeš et al., 2002). Thus, if the classification of all these species is correct, pro-kDNA is found in each of the three major bodonid clades. However, it is not possible to reconstruct the evolutionary history of $\mathrm{kDNA}$, as the branching orders within the bodonids are uncertain. The prokinetoplastid Ichthyobodo necator contains poly-kDNA and its close relative Perkinsiella-like also contains kDNA that resembles poly-kDNA, although it may be a novel type of kDNA (Dyková et al., 2003). Thus, it can be speculated that the ancestral bodonid also had kDNA of this type. The occurrence of poly-kDNA and pro-kDNA in neobodonids and of pankinetoplast DNA (pan-kDNA) and pro-kDNA in parabodonids (eubodonids probably only contain prokDNA and trypanosomes contain either a conventional kDNA network or mega-kDNA; Lukeš et al., 2002) suggests that the same type of kDNA has evolved multiple times in the history of DNA but, without better-resolved phylogenetic trees, it is not possible to represent its evolution accurately.

In addition to the kDNA type, other key ultrastructural characters of the novel isolate are also not in agreement with the diagnosis of the genera Rhynchobodo and Dimastigella. The isolate lacks extrusomes, characteristic of the genus Rhynchobodo (Brugerolle, 1985), and can also be distinguished easily from the genus Dimastigella, which is characterized by spindle-shaped cells and a recurrent flagellum adhering to a ventral furrow (Vickerman, 1976; Breunig et al., 1993) (Fig. 1d).

The genus Rhynchomonas, which may contain the same kDNA type as the novel isolate, is characterized by a significantly different ultrastructure. A proboscis (a large anterior hollow and flexible process considered to be unique among kinetoplastids) attached along the length of the short anterior flagellum, the angle of the flagellar bases to each other $\left(45^{\circ}\right)$, two hair-bearing flagella and a hollow canal (extending proboscis) along the entire length of the cell parallel to the dorsal surface (Larsen \& Patterson 1990; Swale, 1973; Vickerman, 2000a) are among a selection of characters that distinguishes the genus Rhynchomonas from the novel isolate.

However, the case of the remaining genus, Neobodo, does not seem nearly as clear as with the other four genera. In most respects, the novel isolate is identical to the genus Neobodo (Moreira et al., 2004), but it differs in one criterion. Members of the genus Neobodo have a prismatic rod of microtubules that supports the apical cytostome and cytopharynx (Eyden, 1977; Moreira et al., 2004). The novel isolate also possesses such microtubules; however, they were clearly not prismatic in any of the TEM sections (Fig. 3h). Based on the molecular analysis, together with the ultrastructural diagnosis, we suggest that the novel isolate represents a novel genus within the order Neobodonida, Actuariola gen. nov. As kinetoplastids are small organisms, there may be limitations in terms of ultrastructuralmorphological characters that are able to distinguish between species or even genera. The small size and lack of characteristic ultrastructural details is a well-known restriction for small protists when it comes to taxonomic identification (Eyden, 1977; Larsen \& Patterson, 1990; Tong, 1997; von der Heyden, 2004). Thus, in most cases, molecular data may be a powerful tool to complete morphologicalultrastructural studies. The resolving power of $18 \mathrm{~S}$ rDNA analysis for kinetoplastid flagellates was demonstrated only recently (Moreira et al., 2004; Simpson et al., 2002; von der Heyden et al., 2004; von der Heyden, 2004).

Regarding the isolate Cryptaulaxoides-like sp. TCS2003, von der Heyden et al. (2004) mentioned (the appearance of) a spiral groove, characteristic of the genus Cryptaulax Skuja 1948 (=Cryptaulaxoides Novarino 1996). However, using SEM, TEM and live observation, we were not able to confirm the existence of a spiral groove on our isolate. In addition, all supposed Cryptaulax species were assigned to the genera Rhynchobodo and Hemistasia (Bernard et al., 2000). Similar to the isolate described in this study, members of the genus Hemistasia do not have a spiral groove (Elbrächter et al., 1996). However, Elbrächter et al. (1996) describe Hemistasia as polykinetoplastic, lacking a distinct rod organ and discharging extrusomes of the lattice tube type, which clearly distinguishes FV18-8TS from Hemistasia. Thus, there is no evidence for the described sequence clade being assigned either to the disputed genus Cryptaulax or to the genus Hemistasia.

\section{Ecology}

The cells move in two ways, by creeping along the substratum or by swimming freely in the medium. Freeswimming cells usually have straightforward motion and turn in a right spiral around their body axes. Whilst swimming, the recurrent flagellum is wrapped around the body $(0 \cdot 5-0 \cdot 75$ right turns). In some cells, swimming sometimes appears to be inefficient, i.e. a jerking and wobbly progression only (cf. Rhynchomonas Swale 1973). The general behaviour of $A$. framvarensis when creeping was similar to that of Rhynchomonas nasuta (cf. Swale, 1973). Creeping cells move rapidly and smoothly forward. The longer flagellum trails behind, whereas the shorter flagellum is directed forward. The recurrent flagellum is free from the body. On contact with particles, especially food particles, the beating of the short flagellum is interrupted and the particle is handled as described for Rhynchomonas (cf. Boenigk \& Arndt, 2000). Flagellates can also attach to the substratum by means of the recurrent flagellum. When attached, they may make sudden and rapid jerks by bending the posterior flagellum. The cells are attracted by bacterial aggregations and accumulate at such spots. Similar to other substratebound bodonids, the investigated flagellate fed preferentially on substrate-bound bacteria (Caron, 1987).

A. framvarensis was isolated from the oxic-anoxic interface of the Framvaren Fjord in southern Norway. Ecophysiological experiments demonstrated that suboxic conditions, even though they may not be optimal, provide an alternative habitat for the organism. However, it does not tolerate 
strictly anoxic conditions (Fig. 4a). One mechanism for surviving anoxic conditions is the formation of cysts (Fig. 1g, h). Sequence AT5-25, a probable member of the proposed genus Actuariola, was discovered in an oxygendepleted marine hydrothermal vent environment (LópezGarcía et al., 2003). Bodonids in general have a widespread ability to survive under anaerobic and suboxic conditions

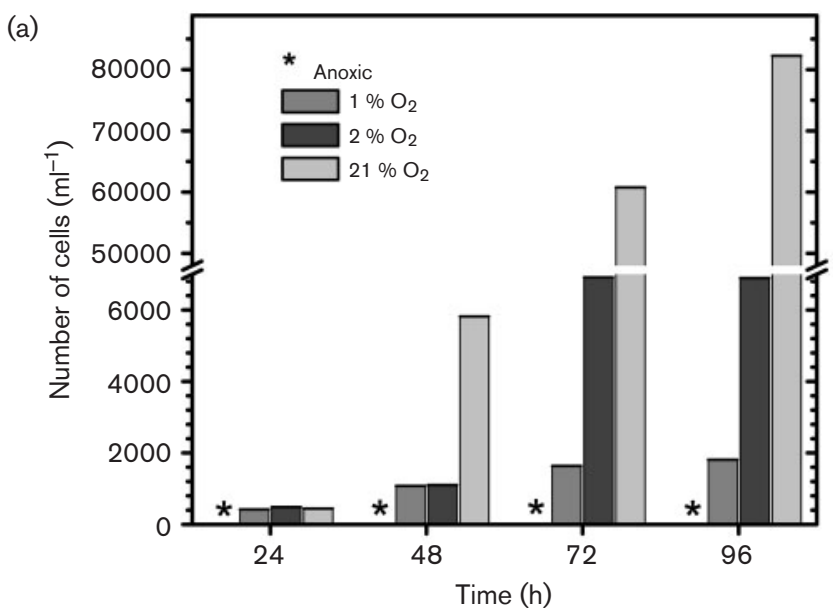

(b)

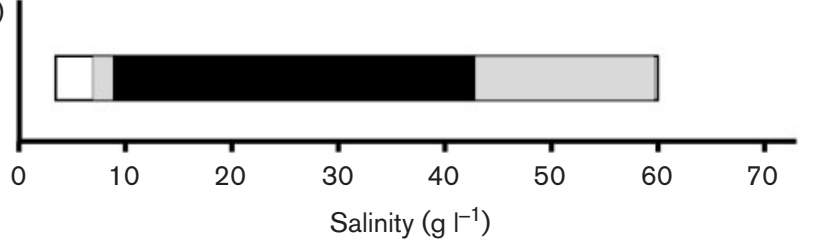

(c)

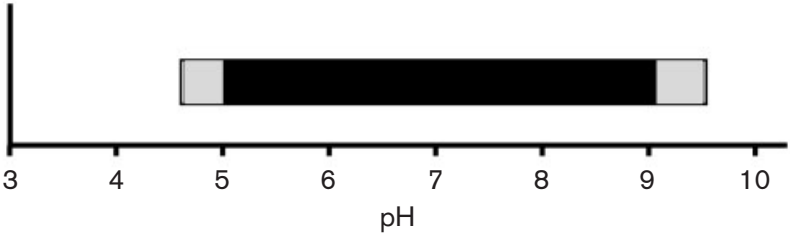

(d)



Fig. 4. Ecophysiological tolerance limits for $A$. framvarensis. (a) Oxygen. The number of cells was counted for $96 \mathrm{~h}$ under foodsaturated conditions and anoxic, suboxic (1 and $2 \% \mathrm{O}_{2}$ ) and oxygen-saturated $\left(21 \% \mathrm{O}_{2}\right)$ conditions. No active cells were found under anoxic conditions. (b)-(d) Salinity (b), pH (c) and temperature (d). Filled bars, growth after direct transfer; shaded bars, survival but no significant growth; and open bars, survival after stepwise adaptation. Apart from low salinity, adaptation did not significantly alter the tolerance limits. As measurement of the critical minimal temperature for growth is difficult, the threshold for a growth rate of less than $0 \cdot 1$ day $^{-1}$ is given (hatched bar). The lowest temperature investigated was $4{ }^{\circ} \mathrm{C}$.
(Bernard et al., 2000); however, as yet, nothing is known about the metabolism of free-living bodonids (Vickerman, $2000 \mathrm{~b})$. The possession of peroxisome-like structures (Fig. 3c) and the RNA-editing capability of the kinetoplast of free-living bodonids (Blom et al., 1998) are links to a lifestyle as wanderers between oxic and anoxic worlds (Cavalier-Smith, 1997). The advantages of such a lifestyle could be a greater abundance of food organisms at the oxicanoxic interface together with a decrease in predation pressure, as predators are less abundant (Fenchel \& Finlay, 1995). However, because of a reduced energy metabolism under anoxic conditions, growth rates are slower.

The tolerance limits of $A$. framvarensis cover the range of temperate marine and brackish waters (Fig. 4). Surprisingly, A. framvarensis did not survive at temperatures below $6-8^{\circ} \mathrm{C}$ (Fig. 4d). Again, cyst formation may be a suitable adaptation to survive seasons of unfavourable conditions. However, as we tested tolerance limits only, we could not determine the actual niche of the flagellate, which must be assumed to be much narrower. With a FISH probe, we provide a tool to access the target organism in nature and study its distribution and ecological niche (Fig. 5). The highest hybridization stringency occurred between 40 and $50 \%$ formamide (Fig. 5). Future research with $A$. framvarensis will focus on physiological and ecological experiments, and on in situ studies using the FISH probe to explore the organism's metabolism and role in natural systems, thus providing the first detailed ecological examination of a freeliving bodonid.

\section{Taxonomic appendix}

\section{Genus: Actuariola gen. nov.}

Diagnosis. Solitary phagotrophic flagellate with a single stainable, discrete prokinetoplast (pro-kDNA). Recurrent flagellum free or mainly free from body. Cystostome apical. Cystostome-cytopharynx supported by a nonprismatic rod of microtubules. Type species, Actuariola framvarensis.

Remarks. Resembles the genus Neobodo, but differs from it in the arrangement of the rod of microtubules that supports the cytopharynx and the cytostome.

Etymology. Actuariola is the Latin name for boat, ship's boat, shallop or sloop, dating back in their basic design to the early Viking vessels. The body outline (side view) of our isolate resembles a shallop's hull. The species name, Actuariola framvarensis, is attributed to the location from which the organism was isolated (Framvaren fjord, Norway).

\section{Species: Actuariola framvarensis sp. nov.}

Extended diagnosis. Cells are elongated, highly active, with two heterodynamic, subapically inserted flagella, a rounded posterior end and an asymmetric apex with a 
T. Stoeck and others

(a)

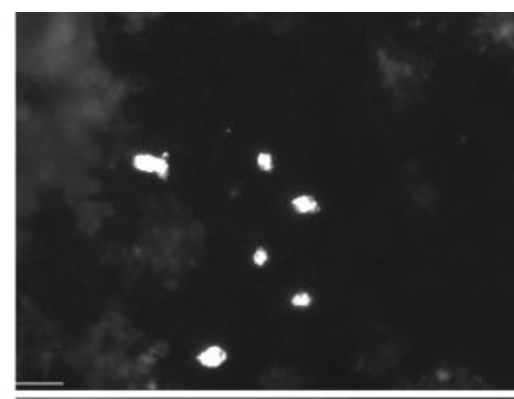

(b)

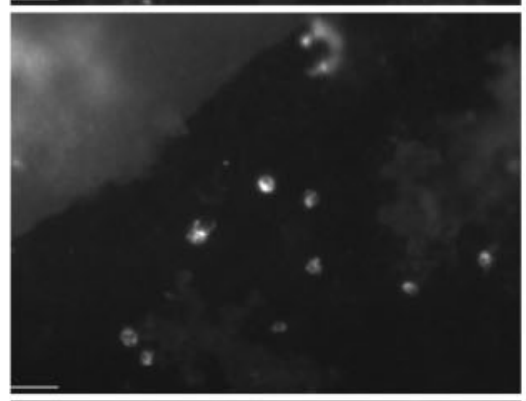

(c)

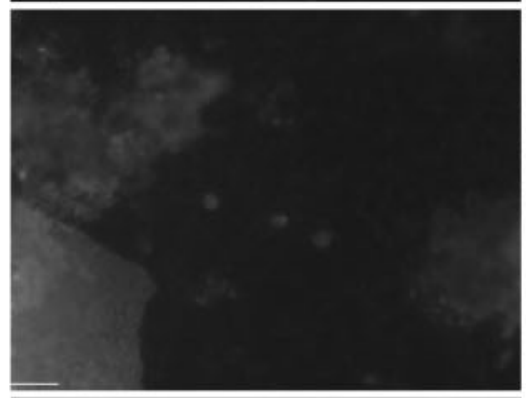

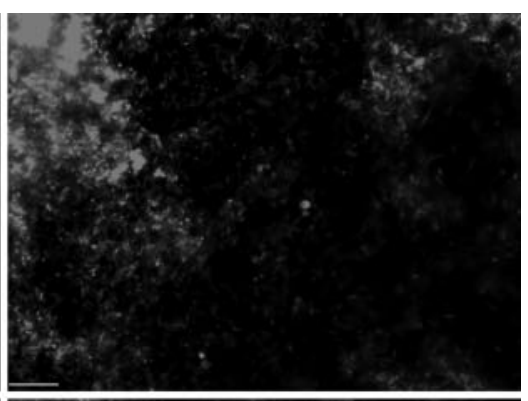


(d)

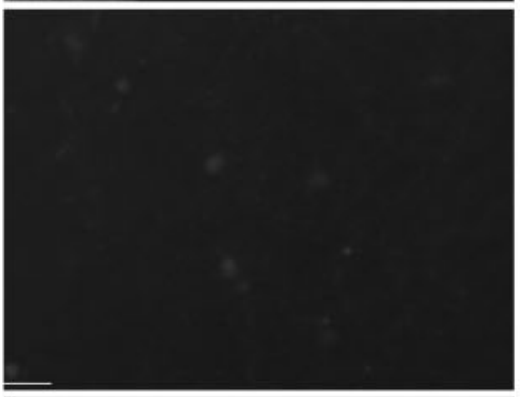

(e)

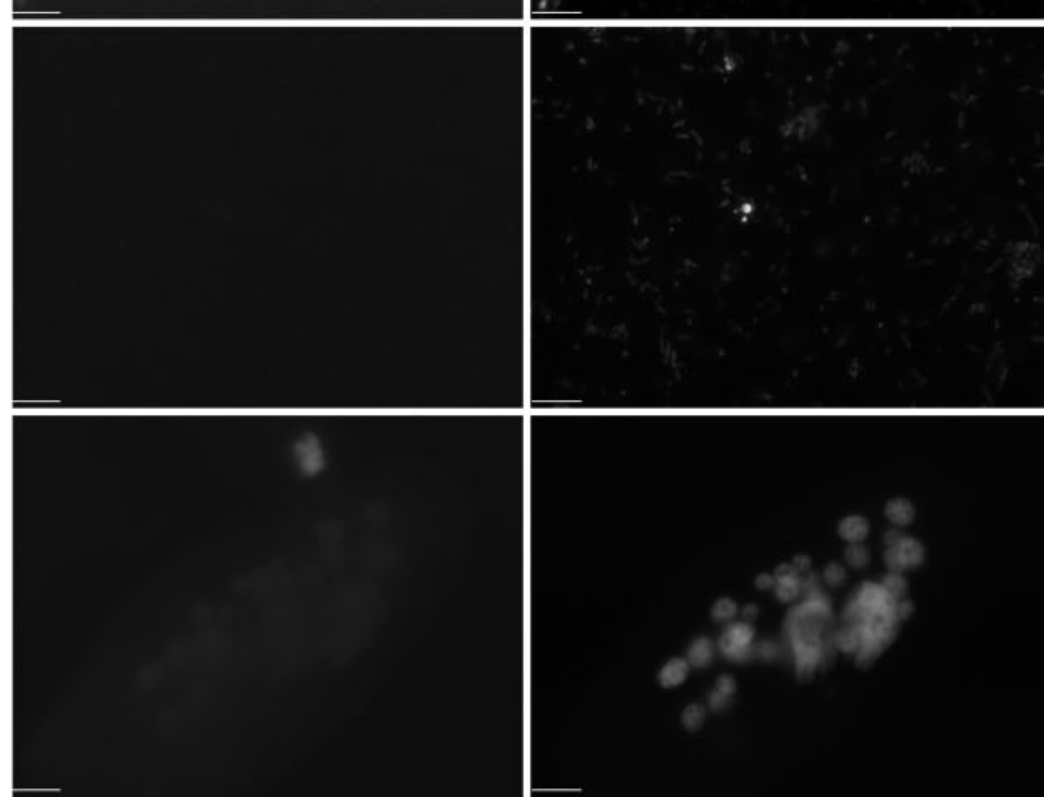

Fig. 5. FISH. Left panels, formamide (FA) concentration series and controls. Right panels, DAPI counterstaining to visualize nuclei and kinetoplasts of the target cells. (a) A. framvarensis with probe FV18TS-650 hybridized at $40 \% \mathrm{FA}$, resulting in a very strong signal. (b) A. framvarensis with probe FV18TS-650 hybridized at 50\% FA, resulting in a clear signal compared with the controls. (c) A. framvarensis with probe FV18TS-650 hybridized at $60 \%$ FA, resulting in a hardly detectable signal. (d) $A$. framvarensis with probe FV18TS-650 hybridized at $70 \%$ FA. There was no difference between $70 \%$ FA and the non-target cell hybridization. (e) Negative control. No signal was obtained with a non-probe sample of $A$. framvarensis that was treated the same as all of the other samples. (f) Non-target cell hybridization; typical negative signal for $P$. caudatum hybridized with probe FV18TS650 at $40 \%$ FA. Additional FA concentretions and non-target organisms were tested, but are not shown. During all FISH analyses and image captures, the microscope and camera settings were constant throughout the experiment. Bars, $5 \mu \mathrm{m}$.

2632

International Journal of Systematic and Evolutionary Microbiology 55 
short rostrum (Fig. 1a-d). The surface of the cell is smooth. The mean body length of living cells $(n=66)$ in the exponential growth phase is $7 \cdot 33 \pm 1 \cdot 19 \mu \mathrm{m}( \pm \mathrm{SD})$, the mean body width is $2 \cdot 46 \pm 0 \cdot 37 \mu \mathrm{m}$ and the ratio of body length to width is $2 \cdot 97$. The two flagella emerge in a deep subapical flagellar pocket; one anterior and the other posterior. They are unequal in length and leave the flagellar pocket at an angle of about $90^{\circ}$ to each other (Figs la, $\mathrm{b}, \mathrm{f}$ and $\mathrm{ee}$ ). The anterior flagellum is approximately twice the body length, whereas the acronematic recurrent flagellum is approximately four times the body length (Figs 1c, $\mathrm{f}$ and $3 \mathrm{~b}$ ). The acroneme, approximately $5 \mu \mathrm{m}$ long, is not visible with light microscopy of living cells. The two flagella do not possess mastigonemes and have a paraxial rod at least at the base (Fig. 3d, f). A surface coat of the anterior flagellum is missing, but microfilaments are present at the flagellar membrane of the recurrent flagellum, restricted to an area within the flagellar pocket (Fig. 3e). The recurrent flagellum is not attached to the plasma membrane of the flagellar groove outlining the position of the posterior flagellum alongside the cell body. The transition zone of the basal bodies displays a transverse plate (Fig. 3c, e). The origin of the axoneme in both flagella is at this plate where no helical structures are associated. A ventral view of the cell shows the depth of the flagellar pocket and that the rostrum at the apical end is relatively short (Fig. 1d). The cytostome is located apically inside the rostrum and is surrounded by lappets (Fig. 3a, d). Inside the rostrum, microtubules are arranged in a longitudinal and circumpolar arrangement. A juxta-pharyngeal band of microtubules is associated with the tubular cytostome (Fig. 3f). The cytostome leads to a long tubular cytopharynx supported by an rod organ until its final end posteriorly to the mitochondrion (Fig. 3f). Both the cytopharynx and the associated rod organ are arranged in a hook-like manner, outlining the posterior side of the single mitochondrion (Fig. 3f). The rod organ displays a non-prismatic (Fig. 3h) arrangement in cross-section. The large, single mitochondrion with plate-like cristae contains a single large, stainable kinetoplast, with loosely arranged fibrils (pro-kDNA type; Figs 1e and $3 \mathrm{c}, \mathrm{f}, \mathrm{g}$ ). Basal bodies are directly connected to the apical end of the mitochondrion. Peroxisome-like bodies are found close to the mitochondrion and the nucleus (Fig. 3c). The ovoid nucleus with a central nucleolus is located at the lower end of the flagellar pocket and its chromatin is arranged at the periphery, attached to the nuclear membrane (Fig. 3a, g).

The flagellate feeds on bacteria and forms antapically one large food vacuole that occupies the posterior third to half of the cell body (Fig. 1b and Fig. 3a). Intracytoplasmic bacteria about $1 \mu \mathrm{m}$ in length and $0 \cdot 2 \mu \mathrm{m}$ in width without peribacterial membranes are present between the nucleus, mitochondria and food vacuoles (Fig. 3a, a', g). The flagellate forms ovoid cysts of $6 \cdot 2 \pm 1 \cdot 5 \mu \mathrm{m}$ in length and $3 \cdot 1 \pm 0 \cdot 6 \mu \mathrm{m}$ in width $(n=21)$ (Fig. $1 \mathrm{~g}, \mathrm{~h})$. A spiral groove is not observed during swimming (slow-motion video stacks), when embedded in low-melting-point agar or in SEM preparations. A contractile vacuole is absent. Growth occurs at salinities between 8.9 and $42.9 \mathrm{~g} \mathrm{l}^{-1}$ (Fig. 4b). Cells survive at lower and higher salinity but no growth occurs, and the organism tolerates temperatures of 6$30 \cdot 5{ }^{\circ} \mathrm{C}$ (Fig. $4 \mathrm{~d}$ ). The flagellate grows well at $\mathrm{pH} 5-9$ and survives at $\mathrm{pH} 4 \cdot 6-9 \cdot 5$ (Fig. 4c). Stepwise acclimatization did not expand the tolerated $\mathrm{pH}$ and temperature ranges, but did extend the salinity range $\left(3 \cdot 8-59 \cdot 9 \mathrm{~g} \mathrm{l}^{-1}\right)$. Grows very well under oxygen saturation $\left(\mu_{\max } 3 \cdot 1 \mathrm{day}^{-1}\right.$ at $16^{\circ} \mathrm{C}$ at saturated food concentration). Under micro-oxic conditions, the organism has a decreased growth rate and forms cysts under anoxic conditions (Fig. 1g, h, Fig. 4a).

\section{ACKNOWLEDGEMENTS}

We would like to thank the crew of Nordstranda (Helvik, Norway) for their hospitality during field work in the Framvaren Fjord and H.-W. Breiner, the captain of our research vessel. We thank Karolina Kolodziej for probe optimization and evaluation (FISH), Ed Jarrol (Marine Science Center of Northeastern University, Nahant, USA) and two anonymous reviewers for helpful comments on the manuscript. This study was funded by a grant from the Deutsche Forschungsgemeinschaft (DFG) to T. S. (STO414/2-2).

\section{REFERENCES}

Altschul, S. F., Madden, T. L., Schäffer, A. A., Zhang, J., Zhang, Z., Miller, W. \& Lipman, D. J. (1997). Gapped BLAST and PSI-BLAST: a new generation of protein database search programs. Nucleic Acids Res 25, 3389-3402.

Amaral Zettler, L. A., Gomez, F., Zettler, E., Keenan, B. G., Amils, R. \& Sogin, M. L. (2002). Eukaryotic diversity in Spain's River of Fire. Nature 417, 137.

Arndt, H., Dietrich, D., Auer, B., Cleven, E., Grafenham, T., Weitere, M. \& Mylnikov, A. P. (2000). Functional diversity of heterotrophic flagellates in aquatic ecosystems. In The Flagellates: Unity, Diversity and Evolution, pp. 240-268. Edited by B. S. C. Leadbeater \& J. C. Green. London: Taylor \& Francis.

Bernard, C., Simpson, A. G. B. \& Patterson, D. J. (2000). Some free-living flagellates (Protista) from anoxic habitats. Ophelia 52, 113-142.

Berney, C., Fahrni, J. \& Pawlowski, J. (2004). How many novel eukaryotic 'kingdoms'? Pitfalls and limitations of environmental DNA surveys. BMC Biol 2, 13. http://www.biomedcentral.com/17417007/2/13

Blom, D., de Haan, A., van den Berg, M., Sloof, P., Jirků, M., Lukeš, J. \& Benne, R. (1998). RNA editing in the free-living bodonid Bodo saltans. Nucleic Acids Res 26, 1205-1213.

Boenigk, J. \& Arndt, H. (2000). Comparative studies on the feeding behavior of two heterotrophic nanoflagellates: the filter-feeding choanoflagellate Monosiga ovata and the raptorial-feeding kinetoplastid Rhynchomonas nasuta. Aquat Microb Ecol 22, 243-249.

Boenigk, J., Pfandl, K., Stadler, P. \& Chatzinotas, A. (2005). High diversity of the 'Spumella-like' flagellates: an investigation based on the SSU rRNA gene sequences of isolates from habitats located in six different geographic regions. Environ Microbiol 7, 685-697.

Breunig, A., Köning, H., Brugerolle, G., Vickerman, K. \& Hertel, H. (1993). Isolation and ultrastructural features of a new strain of 
Dimastigella trypaniformis Sandon 1928 (Bodonina, Kinetoplastida) and comparison with a previously isolated strain. Eur J Protistol 29, 416-424.

Brugerolle, G. (1985). Des trichocystes chez les bodonides, un caractère phylogénétique supplémentaire entre Kinetoplastida et Euglenida. Protistologica 21, 339-348 (in French).

Callahan, H. A., Litaker, R. W. \& Noga, E. J. (2002). Molecular taxonomy of the suborder Bodonina (Order Kinetoplastida), including the important fish parasite, Ichthyobodo necator. J Eukaryot Microbiol 49, 119-128.

Caron, D. A. (1987). Grazing of attached bacteria by heterotrophic microflagellates. Microb Ecol 13, 203-218.

Cavalier-Smith, T. (1981). Eukaryote kingdoms: seven or nine? Biosystems 14, 461-481.

Cavalier-Smith, T. (1997). Cell and genome coevolution: facultative anaerobiosis, glycosomes and kinetoplastan RNA editing. Trends Genet 13, 6-9.

Cavalier-Smith, T. (2004). Only six kingdoms of life. Proc Biol Sci 271, 1251-1262.

Dawson, S. C. \& Pace, N. R. (2002). Novel kingdom-level eukaryotic diversity in anoxic environments. Proc Natl Acad Sci U S A 99, 8324-8329.

de Faria, J. G., Cunha, A. M. \& Pinto, C. (1922). Estudos sobre protozoairos do mar. Mem Inst Oswaldo Cruz 15, 186-208 (in Spanish).

Del Giorgio, P. A., Gasol, J. M., Vaque, D., Mura, P., Agusti, S. \& Duarte, C. M. (1996). Bacterioplankton community structure: protists control net production and the proportion of active bacteria in a coastal marine community. Limnol Oceanogr 41, 1169-1179.

Doležel, D., Jirků, M., Maslov, D. A. \& Lukeš, J. (2000). Phylogeny of the bodonid flagellates (Kinetoplastida) based on small-subunit rRNA gene sequences. Int J Syst Evol Microbiol 50, 1943-1951.

Dyková, I., Fiala, I., Lom, J. \& Lukeš, J. (2003). Perkinsiella amoebaelike endosymbionts of Neoparamoeba spp., relatives of the kinetoplastid Ichthyobodo. Eur J Protistol 39, 37-52.

Elbrächter, M., Schnepf, E. \& Balzer, I. (1996). Haemistasia phaeocysticola (Scherffel) comb. nov., redescription of a free-living, marine, kinetoplastid flagellate. Arch Protistenkd 147, 125-136 (in German).

Eyden, B. P. (1977). Morphology and ultrastructure of Bodo designis Skuja 1948. Protistologica 13, 169-179.

Fenchel, T. \& Finlay, B. J. (1995). Ecology and Evolution in Anoxic Worlds. Oxford: Oxford University Press.

Foissner, W. (1991). Diversity and ecology of soil flagellates. In The Biology of Free-Living Heterotrophic Flagellates, pp. 93-112. Edited by D. J. Patterson \& J. Larsen. New York: Clarendon Press.

Frolov, A. O. \& Malysheva, M. N. (2002). Ultrastructure of the flagellate Cruzella marina (Kinetoplastidea). Tsitologiia 44, 447-484 (in Russian).

Hannaert, V., Bringaud, F., Opperdoes, F. R. \& Michels, P. A. M. (2003). Evolution of energy metabolism and its compartmentation in Kinetoplastida. Kinetoplastid Biol Dis 2, 11.

Hahn, M. M., Lünsdorf, H., Wu, Q., Schauer, M., Höfle, M. G., Boenigk, J. \& Stadler, P. (2003). Isolation of novel ultramicrobacteria classified as Actinobacteria from five freshwater habitats in Europe and Asia. Appl Environ Microbiol 69, 1442-1451.

Hollande, A. (1952). Ordre des Bodonides (Bodonidea ord. nov.). In Traité de Zoologie, pp. 669-693. Edited by P. P. Grassé. Paris: Masson \& Cie (in French).

Larsen, J. \& Patterson, D. J. (1990). Some flagellates (Protista) from tropical marine sediments. J Nat Hist 24, 801-937.

López-García, P., Rodriguez-Valera, F., Pedros-Alio, C. \& Moreira, D. (2001). Unexpected diversity of small eukaryotes in deep-sea Antarctic plankton. Nature 409, 603-607.
López-Garcia, P., Philippe, H., Gail, F. \& Moreira, D. (2003). Autochthonous eukaryotic diversity in hydrothermal sediment and experimental microcolonizers at the Mid-Atlantic Ridge. Proc Natl Acad Sci U S A 100, 697-702.

Ludwig, W., Strunk, O., Westram, R. \& 29 other authors (2004). ARB: a software environment for sequence data. Nucleic Acids Res 32, 1363-1371.

Lukeš, J., Guilbride, D. L., Votypka, J., Zíkova, J., Benne, R. \& Englund, P. T. (2002). Kinetoplast DNA network: evolution of an improbable structure. Eukaryot Cell 1, 495-502.

Maddison, D. \& Maddison, W. (2000). MacClade 4: Analysis of Phylogeny and Character Evolution. CD-ROM. Sunderland, MA: Sinauer Associates.

Moreira, D., López-García, P. \& Vickerman, K. (2004). An updated view of kinetoplastid phylogeny using environmental sequences and a closer outgroup: proposal for a new classification of the class Kinetoplastea. Int J Syst Evol Microbiol 54, 1861-1875.

Novarino, G. (1996). Notes on flagellate nomenclature. I. Cryptaulaxoides nom. n., a zoological substitute for Cryptaulax Skuja, 1948 (Protista incertae sedis) non Cryptaulax Tate, 1869 (Mollusca, Gastropoda) non Cryptaulax Cameron (Insecta, Hymenoptera), with remarks on botanical nomenclature. Acta Protozool 35, 235-238.

Pernthaler, J., Gloeckner, F. O., Schoenhuber, W. \& Amann, R. (2001). Fluorescence in situ hybridization (FISH) with rRNAtargeted oligonucleotide probes. Methods Microbiol 30, 207-226.

Posada, D. \& Crandall, K. A. (1998). MODELTEST: testing the model of DNA substitution. Bioinformatics 14, 817-818.

Reize, I. B. \& Melkonian, M. (1989). A new way to investigate living flagellated/ciliated cells in the light microscope: immobilization of cells in agarose. Bot Acta 102, 145-151.

Reynolds, E. S. (1963). The use of lead citrate at high $\mathrm{pH}$ as an electronopaque stain in electron microscopy. J Cell Biol 17, 208-212.

Simpson, A. G., Lukeš, J. \& Roger, A. J. (2002). The evolutionary history of kinetoplastids and their kinetoplasts. Mol Biol Evol 19, 2071-2083.

Skei, J. M. (1988). Framvaren - environmental settings. Mar Chem 23, 209-218.

Spurr, A. R. (1969). A low-viscosity epoxy resin embedding medium for electron microscopy. J Ultrastruct Res 26, 31-43.

Stoeck, T., Taylor, G. T. \& Epstein, S. S. (2003a). Novel eukaryotes from the permanently anoxic Cariaco Basin (Caribbean Sea). Appl Environ Microbiol 69, 5656-5663.

Stoeck, T., Fowle, W. H. \& Epstein, S. S. (2003b). Methodology of protistan discovery: from rRNA detection to quality scanning electron microscope images. Appl Environ Microbiol 69, 6856-6863. Swale, E. M. F. (1973). A study of the colourless flagellate Rhynchomonas nasuta (Stokes) Klebs. Biol J Linn Soc 5, 255-264.

Swofford, D. L. (2001). PAUP ${ }^{\star}$ - Phylogenetic Analysis Using Parsimony (*and other methods), v. 4.0b6. Sunderland, MA: Sinauer Associates.

Thompson, J. D., Higgins, D. G. \& Gibson, T. J. (1994). CLUSTAL W: improving the sensitivity of progressive multiple sequence alignment through sequence weighting, position-specific gap penalties and weight matrix choice. Nucleic Acids Res 22, 4673-4680.

Tong, S. (1997). Heterotrophic flagellates and other protists from Southampton water, UK. Ophelia 47, 71-131.

Vickerman, K. (1976). The diversity of the kinetoplastid flagellates. In Biology of the Kinetoplastida, pp. 1-34. Edited by W. H. R. Lumsden \& D. A. Evans. London: Academic Press.

Vickerman, K. (2000a). Order Kinetoplastea. In The Illustrated Guide to the Protozoa, pp. 1159-1185. Edited by J. J. Lee, G. F. Leedale \& P. Bradbury. Lawrence, KS: Allen Press. 
Vickerman, K. (2000b). Adaptations to parasitism. In The Flagellates: Unity, Diversity and Evolution, pp. 190-216. Edited by B. S. C. Leadbeater \& J. C. Green. London: Taylor \& Francis.

von der Heyden, S. (2004). Testing ubiquitous dispersal and freshwater/marine divergence in free-living protist groups. $\mathrm{PhD}$ thesis, University of Oxford.

von der Heyden, S., Chao, E. E., Vickerman, K. \& Cavalier-Smith, T.

(2004). Ribosomal RNA phylogeny of bodonid and diplonemid flagellates and the evolution of Euglenozoa. J Eukaryot Microbiol 51, 402-416.

Vørs, N. (1992). Heterotrophic amoebae, flagellates and heliozoa from the Tvärminne area, Gulf of Finland, in 1988-1990. Ophelia 36, $1-109$.

Willerslev, E., Hansen, A. J., Christensen, B., Steffensen, J. P. \& Arctander, P. (1999). Diversity of Holocene life forms in fossil glacier ice. Proc Natl Acad Sci U S A 96, 8017-8021. 